\title{
LAS AUCTORITATES BÍBLICAS EN RAMON LLULL: ETAPA 1304-1311
}

\author{
Jordi Pardo Pastor \\ Archivivm Lvllianvm-Universitat Autònoma de Barcelona \\ Instituto Brasileiro de Filosofia e Ciência Raimundo Lúlio («Ramon Llul»)
}

\begin{abstract}
RESUMEN
La originalidad del sistema luliano de no argumentar mediante autoridades incluye en dicha perspectiva la Biblia, pues en la Edad Media cristiana la autoridad más importante proviene de las sagradas escrituras. En este punto de vista, Ramon Llull adopta una peculiar visión respecto a la Biblia: si el Ars luliana es un sistema que va más allá de la Biblia, la Biblia es uno de los argumentos para ir más allá de Ella misma. En este caso, la Biblia y, especialmente, algunas citas bíblicas son la estructura básica del concepto apostólico del sistema luliano.
\end{abstract}

Palabras clave: Ramon Llull, Biblia, autoridades.

\begin{abstract}
The originality of the lullian system of not arguing with authorities includes also the Holy Bible, because in the Christian Middle Ages the most important authority is the holy word. In this context, Ramon Llull adopts a especial vision with reference to the Holy Bible: if the lullian Ars is a system which goes further than the Bible, then the Holy Bible itself is one of the arguments for going much further than the Bible. In this case, the Holy Bible and, especially, some of the biblical quotations are the basic structure of the apostolical conception of the lullian system.
\end{abstract}

Key words: Ramon Llull, Bible, authorities.

\section{INTRODUCTIO GENERALIS}

Los inventarios de la época altomedieval catalana hablan por sí mismos en cuanto a los fondos de libros que se conservan tanto en bibliotecas institucionales como privadas. ${ }^{\prime}$ Previamente a la revolución cultural que se produce entre los siglos XI y XII, la principal ocupación de los hombres cultos consistía en interpretar convenientemente la ley divina, estableciéndose como objetivo primordial el conocer a la perfección la Biblia y transmitir su mensaje al pueblo mediante la predicación. De ello, se alcanza la obligada lectura de los santos padres y libros litúrgicos que recreaban y desplegaban el culto a Dios. Sin embargo, es difícil contar con Biblias completas ya en bibliotecas monásticas, catedralicias y parroquiales, ya en bibliotecas

1 Sigo el libro de Jesús Alturo i Perucho, Història del llibre manuscrit a Catalunya, Generalitat de Catalunya. Entitat Autònoma del Diari Oficial i de Publicacions, 2003, esp. pp. 69-227. 
privadas de obispos, canónigos, sacerdotes rurales o personajes laicos, por el elevado precio que éstas adquirían. Con todo, el que fuera difícil no significa que no las hubiera. ${ }^{2}$

El camino de los hombres cultos del Doscientos seguirá andándose por la lectura de la $\mathrm{Bi}$ blia - en ocasiones de forma incompleta, leyendo, sólo, los libros que se encontraban a su disposición-, de la patrística y obras que comentan las fuentes bíblicas, naciendo las auctoritates. El crecimiento de la producción literaria, que no cesó de aumentar a partir del siglo XII, condujo a tomar «otros métodos de lectura más rápidos que les permitieran a los intelectuales adquirir el conocimiento de gran número de obras». ${ }^{3}$ La costumbre escolástica de citar auctoritates llevó a la composición de florilegios o compilaciones de textos para ser memorizados y que habilitaban a encontrar con facilidad los pasajes buscados. Estos florilegios servían, a su vez, como cuaderno de sentencias que podían citarse en las obras medievales, reforzando, así, una idea propia del autor. Por ello, es usual hallar en las obras escolásticas un sin fin de citas bíblicas y patrísticas que, de seguro, no provienen de un conocimiento expreso a raíz de una asimilación y completa lectura de una determinada doctrina expuesta en un texto del momento. Hamesse propone dos formas de acudir a la auctoritas: mediante estos mencionados florilegios —que llevaban por asociación al texto original — y/o mediante una lectura fragmentaria centrada en fragmentos escogidos, ambas a la disposición de la utilidad.

A excepción del:modus operandi de todos los autores escolásticos, Ramon Llull (12321316) crea un método que sectores de la crítica han denominado como autoridad alternativa. ${ }^{4}$ El ideal luliano se basa en tres puntos: 1) la conversión del infiel y los incrédulos a la santa fe católica, y para ello poner en peligro de muerte la propia vida; 2) escribir unum librum meliorem de mundo contra los errores de los infieles; y 3) la creación de monasterios para que se formaran hombres doctos y sabios en la cultura y lengua árabes con una clara finalidad: que éstos pudieran predicar a los infieles la santa fe católica. ${ }^{5} \mathrm{~A}$ la par, este ideario de predicación al infiel se define en los siguientes párrafos que transcribimos a modo de ejemplo:

Infideles non stant ad auctoritates fidelium, et tamen stant ad rationes. Et sic de multis aliis rationibus, quae dici possent. Per quas monstratur, quod iste tractatus non est contra fidem, sed est ad exaltationem et honorem fidei christianae. Quoniam infideles dicunt: Nolumus dimittere fidem pro fide aut credere pro credere; sed bene credere pro intelligere dimittemus. ${ }^{6}$

Et etiam conuerunt inter se, quod disputarent per modum intelligendi, non per auctoritates, quoniam intelligere est actus primitiuus, uerus et necessarius ipsius, et infallibilis, dum est practicus. Per auctoritates autem intellectus quandoque uadit per credere, quandoque per intelligere, quia sensus auctoritatum patitur mutationem et opiniones.?

2 Véase el resumen que da el profesor Alturo en Història del llibre manuscrit a Catalunya, op. cit. (cfr. nota 1), pp. 96-114, donde menciona los inventarios de diferentes bibliotecas y, especialmente p. 101, donde menciona que el monasterio de Sant Serni de Tavèrnoles poseía dos Biblias completas. Téngase en cuenta, también, que ya en el siglo XIII la Universidad de París comienza una ardua labor de corrección y edición de la Biblia, hecho que permite, a partir de este momento, contar con un mayor grupo de ejemplares. Véase, Samuel Berger, «Des essais qui ont été faits a Paris au treizième siècle. Pour corriger le texte de la Vulgate», Revue de théologie et de philosophie, 16 (1883), Lausanne, pp. 1-26.

3 Jacqueline Hamesse, «El modelo escolástico de la lectura», en Guglielmo Cavallo y Roger Chartier (dir.), Historia de la lectura en el mundo occidental, Madrid, Taurus, 2001, p. 187.

4 Véase, Anthony Bonner, «L'Art lul-liana com a autoritat alternativa», Studia Lulliana, 33/1, (1993 [1994]), pp. 15-32. Véase también $\dot{I} d$., «L'Art de Ramon Llull com a sistema lògic», Randa, 19, Barcelona 1986; Id., «La logique dans l'Art de Raymonde Lulle», Cahiers de Fanjeaux, 22 (1987), pp. 030-305

5 Sebastián Garcías Palou, El Miramar de Ramon Llull, Palma de Mallorca, 1977.

6 Raimundus Lullus, Liber de demonstratione per aequiparantiam (= Raimundi Lulli Opera latina. Tomus VI. Op. lat. 121), Helmut Riedlinger (ed.), (Corpus Christianorum. Continuatio Mediaeualis XXXIII), Turnhout, Brepols, 1978, lin. 116-122.

7 Raimundus Lullus, Disputatio Raimundi et Auerroistae (= Raimundi Lulli Opera latina. Tomus VI. Op. lat. 168), Hermogenes Harada (ed.), (Corpus Christianorum. Continuatio Mediacualis XXXII), Turnhout, Brepols, 1975 , pp. $9-10$. 
Así pues, nace en la apologética 'luliana la necesidad de otorgar rationes necessariae al infiel para convencerlo del error en el cual se halla e inclinarlo a la fe verdadera, no mediante auctoritates, sino mediante una «teología positiva» que conduce a una aequiparantia entre la filosofia y la teología cristianas. ${ }^{8}$ No obstante, el hecho de no basar los argumentos en citas de autoridades en el momento de la predicación al infiel crea un resultado paradójico, pues «al querer prescindir de la Biblia misma, [Llull] prescinde del testimonio de la revelación de una fe revelada. Es curioso también que nos presente [...] una religión casi sin santos, sin historias bíblicas y sin todo el aparato de la Iglesia [...]».9

Ciertamente, Llull no es un autor prolijo en cuanto a citas de autoridades, tales como la Biblia o los padres de la iglesia, aunque sí hallamos, en cierta medida, citas bíblicas en la obra luliana. Por otro lado, Ramon no toma historias de santos o historias de la Biblia, pues este tema queda cubierto por los toques autobiográficos que Llull presenta de sí mismo en sus obras, entendiéndose estos 'microrelatos' como elementos hagiográficos que, simultáneamente, delinean una sutil delimitación entre el santo y el hombre, entre el personaje y Ramon. ${ }^{10}$ Determinados sectores de la crítica lulista consideran que esta identificación entre lo hagiográfico y lo autobiográfico obligaría a Lluil a no tomar citas bíblicas, ya que su intención de escribir el mejor libro del mundo, ejemplificado en el Ars luliana, es una actitud de presentarse como un elegido de Dios, tal y como lo fueron Jesucristo o Mahoma. ${ }^{11}$

8 Debemos calzar pies de plomo al definir el término de «teología positiva» y «teólogo positivo» en Ramon Llull, pues ambas expresiones se alejan de lo que, más tarde, motivará esta tendencia metodológica y teológica en el Humanismo. El concepto luliano de «teología positiva» se toma como modo de expresar uno de los aspectos de la teología, afirmando que la ciencia divina es 'positiva' y 'demostrativa', en el sentido que «Theologia positiva està per voluntat, c demostrativa per enteniment» (Ramon Llull, Proverbis de Ramon, Obres de Ramon Llull XIV, cap. 276, p. 301). Sobre este aspecto, sigo a S. Garcias Palou, « ¿Fue Ramon Llull el primero en usar las expresiones "teología positiva" y "teólogo positivo"?», Estudios Lulianos 2 (1958), pp. 187-196. Por otro lado, Charles Lohr («Ramon Llull-teología positiva-árabes», Estudios Lulianos 17 [1973], pp. 113-123) atiende la cuestión desde una posible influencia de la filosofia árabe a esta nueva metodología teológica. En cuanto a la demonstratio per aequiparantiam remito al lector a Jordi Pardo Pastor, «En torno a las rationes necessariae del conocimiento de Dios: de Santo Tomás de Aquino a Ramon Llull», Estudios Eclesiásticos 77 (2002), pp. 461-475; Íd., «Filosofia y Teología en Ramon Llull: la demonstratio per aequiparantiam», Revista Española de Filosofia Medieval 9 (2002), pp. 265 274; trabajos en los que se aporta bibliografia sobre la cuestión.

9 Lola Badia y Anthony Bonner, Ramón Llull: Vida, pensamiento y obra literaria, Barcelona, Quaderns Crema, 1993, p. 74.

10 A este respecto, véase Lola Badia, «Ramon Llull: Autor i personatge», en Aristotellica et Lulliana magistro doctissimo Charles $H$ Lohr septuagesimum annum feliciter agenti dedicata, Fernando Domínguez, Ruedi Imbach, Theodor Pindl y Peter Walter (edd.), eInstrumenta Patristica) XXVI, Steenbrugis, in Abbatia S. Petri, 1995, pp. 355375; $I d$., «La ficción luliana en los orígenes de las letras catalanas», en F. Domínguez y J. Salas (edd), Constantes y fragmentos del pensamiento luliano. Actas del simposio sobre Ramon Llull en Trujillo, 17-20 septiembre 1994, Tübingen, Niemeyer, 1996, pp. 59-76 (aqui p.65). Véase, también, con relación a los elementos hagiográficos Georg Misch, «Die Selbstzeugnisse des Raimund Llull», en Íd. Geschichte der Autobiographie. IV, 1. Dritter Teil: Das Hochmittelalter in der Vollendung, Leo Delfoss (ed.), Frankfurt am Main, 1967, pp. 55-89; y la reseña a Misch que Alexander Fidora firma en Studia Luliana 97 (2001), pp. 165-166.

11 Véase, Fatma Ben Hamamouche, «Ramon Llull y el mundo islámico», Revue d'Histoire Maghrébine (époque moderne et contemporaine) 77-78 (1995), pp. 113-125. El artículo aquí citado es un resumen de la tesis doctoral de la misma autora que trata, un tanto peligrosamente, la relación de Llull con el islam, afirmando que Llull desdeña las auctoritates bíblicas puesto que su intención es asemejarse a Mahoma y aspira presentar al pueblo musulmán una obra en su propia lengua, que represente la palabra de Dios descendida y revelada. A este respecto, Dominique Urvoy («Vues musulmanes sur la personnalité de Ramon Llull», en Studia Lullistica. Miscellania in honorem Sebastiani Garcias Palou, Mallorca, 1989, pp. 115-118), trata la tesis doctoral que da origen al artículo citado, con epítetos más que contundentes: «étonnement et [...] déception» (p. 117). 


\section{RAMON LLULL Y LA BIBLIA}

En orden a todo lo expuesto más arriba, hay un hecho fundamental e innegable: Ramon Llull no construye sus obras al uso de la escolástica de su época, ${ }^{12}$ afirmación que se podría matizar desde una cronología tanto vital como con relación a los textos escritos. Ciertamente, las primeras obras lulianas carecen de todo tipo de referencia a las sagradas escrituras, siendo Llull de lo más parco en referencias bíblicas o patrísticas, y superponiendo la filosofía musulmana a sus propias impresiones tanto preartísticas como artísticas, tras la revelación en el monte de Randa. ${ }^{13}$ Sin embargo, obras como el Libre d'orde de cavalleria (1274-1275), el Blaquerna (1283) o el Fèlix (1288-1289) siguen unos parámetros caballerescoreligiosos basados en la religión cristiana y en autoridades como san Bernardo y su De Laude Nouae Militiae. ${ }^{14}$ Con todo, la primera obra luliana, tras el Compendium logicae Algacelis (1270), que presenta a Ramon Llull en la sociedad intelectual de la época es el Libre de contemplació (1274), en el que podemos observar en germen todas las preceptivas del Beato. ${ }^{15}$ Esta última obra, quizá una de las más importantes en el sistema luliano por ser una obra temprana y tan enciclopédica, muestra los cánones de estos primeros años de producción luliana, en los que se suprimen, prácticamente, las citas bíblicas y patrísticas, sin olvidar, eso sí, la preceptiva cristiana:

On, qui vol, Sènyer, endreçar ni tornar a veritat aquells qui són en error e qui la moral exposició transponen en contrària exposició, cové que s'ajut ab la sobirana exposició entel-lectual la qual és sobre moral; car enaixí com l'enteniment ha vertut que de la moral puig a l'espiritual, entellectual, sobirana exposició, enaixí ha vertut, com és pujat a la sobirana oració e contemplació, que endreç tots aquells qui són errats en la moral exposició. ${ }^{16}$

12 Véase a este respecto, Anthony Bonner, «Ramon Llull i el rebuig de la tradició clàssica i patrística», en $H o-$ menatge a Miquel Dolç. Actes del XII Simposi de la Secció Catalana i I de la Secció Balear de la SEEC. Palma, 1 al 4 de febrer de 1996, Palma de Mallorca, 1997, pp. 373-385; y J. Mª. Ruiz Simon, L'Art de Ramon Llull i la teoria escolàstica de la ciència, Barcelona, Quaderns Crema, 1999.

13 La superposición de la filosofía musulmana en el sistema luliano comprende puntos tan trascentales como el de las dignitates dei, los correlativos lulianos y las razones necesarias. A este respecto, Miguel Cruz Hernández (El pensamiento de Ramon Llull, Valencia, Castalia-Fundación March, 1977, p. 77) presenta un cuadro sobre la comparación entre las dignitates dei y las hadras, tomadas de Ibn 'Arabi, y los sefirots, tomados del Sefer Yetzirah; Dominique Urvoy («La place de Ramon Llull dans la pensée arabe», Catalan Review 4/1-2 [1990], pp. 201-220) expone que la técnica de los correlativos lulianos proviene de «[...] mécanismes particulièrement adaptés à la langue arabe [...]» (p. 214); y Garcías Palou (Ramon Llull y el Islam, Mallorca, 1982, pp. 141-142) afirma que el motivo por el cual Ramon Llull esgrimió 'razones necesarias' en sus diálogos y controversias contra el islam se debe a que el islam mallorquín posee un aspecto más netamente tradicional y racional que, por ejemplo, el islam peninsular.

14 Véase, A. C. R. Oliver, «El Llibre de l'Orde de Cavalleria de Ramón Llull y el De Laude Novae Militiae de San Bernardo"s, Estudios Lulianos, 1958 (2), 175-186. Sobre el ideal caballeresco a lo divino, Jordi Pardo Pastor, «El caballero a lo divino en Ramon Llull: contra el pecado de la lujuria», Revista Mirabilia, 1 (2001), www.revistamirabilia.com. Marc Egea y Ger («Ramon Llull: del combate espiritual al combate por las armas», Revista Agustiniana 125 (2000), pp. 649-667) analiza el Libre d'orde de cavalleria entendiendo que el caballero debe defender la Iglesia, al igual que los clérigos deben defender la fe católica, mediante armas materiales, aunque nunca debe convertir por la fuerza. Egea y Ger aporta pasajes importantes de las mencionadas obras lulianas en los que se funden combate por las armas y combate intelectual, términos que quedan definidos como 'armes materials' $\mathrm{y}$ 'armes espirituals' para la conversión del infiel. Véase, también, M. Sanchis Guarner, «L'ideal cavallerese definit per Ramon Llull», Estudios Lulianos 2 (1958), pp. 37-62.

15 Véase, Jordi Gayà, «Significación y demostración en el Libre de contemplació de Ramon Llull», en Arištotellica et Lulliana magistro et doctissimo Charles H Lohr septuagesimum annum feliciter agenti dedicata, Fernando Domínguez, Ruedi Imbach, Theodor Pindl y Peter Walter (edd.), «Instrumenta Patristica» XXVI, Steenbrugis, in Abbatia S. Petri, 1995, pp. 477-499.

16 Ramon Llull, Llibre de contemplació, en Obres essencials, II, Barcelona, 1960, Cap. 352, p. 1181. 
Otras obras de este primer período, como por ejemplo la Doctrina pueril (1274-1276), el Ars inuentiua (1289) o la Tabula generalis (1294), son, aún, textos que están forjando la filosofia luliana y que siguen, mutatis mutandis, esta preceptiva general luliana de no tomar auctoritates. Según Reinhardt, hay tres fases en la producción luliana: una primera, representada por el Libre de contemplació, donde «legt Llull die Grundlagen, indem er die traditionelle biblische Hermeneutik mit seiner Sicht der Welt verbindet und von daher neu interpretiert»; una segunda, vista desde la óptica de los Artes praedicandi y de los Sermones (sobre los años 13041308), donde hay un importante paso respecto a la Biblia y se toman citas para respaldar la filosofía luliana; y una última fase, en la que «in den späten Artes praedicandi von 1312 und 1313, tritt die Heilige Schrift ganz in den Schatten der Ars. Die Bibel wird jetzt ganz zurückgeführt auf die Prinzipien der Ars $\gg .{ }^{17}$ Con todo, estas fases presentadas por Reinhardt pueden ser un tanto más expansivas o restringidas, a juzgar por algunos otros textos, aunque lo que sí es indiscutible es que hay una serie de obras en la denominada segunda etapa de Llull en las que se toman las auctoritates, ya sea para probar la propia Ars luliana o por otra intención que se nos escapa.

Si tomamos el Arbor scientiae (1295-1296), éste contiene, más bien, un reducido número de citas bíblicas considerando la extensión ciclópea del texto. Siguiendo la delimitación de Reinhardt, esta obra se situaría en una primera etapa de la producción luliana, aunque, podríamos incluirla ya en un periodo en el que Llull utiliza la Biblia en beneficio de su propio Ars. En el Arbor scientiae tan sólo hallamos seis fragmentos en los que se reflejen las sagradas escrituras. ${ }^{18}$ Se cita a Io. 21, 15 en el Arbor apostolicalis (I, lin. 67) - «Et ideo dixit dominus noster Iesus Christus ad beatum Petrum: "Petre, si diligis me, pasce oues meas"»-; en el mismo Árbol (IV, lin. 13) hay la cita «Dilige dominum Deum tuum ex toto corde tuo, et ex omni mente tua, et ex omnibus uiribus tuis», correspondiente al Deut. 6, 5, Mat. 22, 37, Marc. 12, 30 , y Luc. 10, 27, y en la siguiente línea (lin. 14) se cita «Dilige proximum tuum sicut te ipsum», correspondiente al Leu. 19, 18, a Mat. 22, 39 y Marc. 12, 31. De nuevo en el Arbor apostolicalis (IV, lin. $191 \mathrm{y} \mathrm{V}$, lin. 171) descubrimos «Famem me habere uidistis et non saturastis»-correspondiente a Mat. 25, 42-y «Hoc est enim corpus meum» -correspondiente a Mat. 26, 26 y Marc. 14, 22 -; y en el Arbor quaestionalis (V, lin. 1914 y lin. 3325-3326) hallamos «si non creditis, non intelligitis» - correspondiente a Is. 7, 9-y-correspondiente a Ps. 13 , l; y 52, $1-$, respectivamente.

De estas referencias, en diálogo con los siguientes textos que veremos, entresacamos dos cosas: la primera, como apunta Bonner, ${ }^{19}$ es la fuerte intertextualidad bíblica que se desprende de la obra luliana, pues hay un continuo repetir de citas como «ama Déus ton senyor de tot ton cor, de tota ta ànima, de tota ta pensa e totes tes forces»-la misma cita del Arbor apostolicalis (IV, lin. 13), correspondiente al Deut. 6, 5, Mat. 22, 37, Marc. 12, 30, y Luc. 10, 27, ahora en la versión del Llibre de vertuts e pecats ${ }^{20}$ - o el conocido pasaje de Is. 7, 9 que Llull repite incesantemente para justificar su manera de argumentar sine auctoritates, mediante el entendimiento. La segunda, que las auctoritates no se toman en el estricto sentido de justificar una posición, de ilustrar una reflexión confusa o ad pedem litterae, como sentido inequívoco de la sagrada escritura. No, más bien, la Biblia se halla superpuesta a lo que es el Ars lu-

17 Klaus Reinhardt, «Ramón Llull und die Bible», en Aristotellica et Lulliana magistro doctissimo Charles H Lohr septuagesimum annum feliciter agenti dedicata, Fernando Domínguez, Ruedi Imbach, Theodor Pindl y Peter Walter (edd.), «Instrumenta Patristica» XXVI, Steenbrugis, in Abbatia S. Petri, 1995, pp. 311-331, aquí p. 329.

18 Raimundus Lullus, Arbor scientiae (=Raimvndi Lulli Opera Latina. Tomus XXIV-XXV-XXVI. Op. lat. 65), Pere Villalba (ed.), (Corpus Christianorum. Continuatio Mediaeualis, CLXXX A-B-C), vols. I-III (1*-188*+1-1438; 104 ilustraciones), Turnhout, Brepols, 2000.

19 A. Bonner, «L'Art lul-liana com a autoritat alternativa», art. cit., p. 16-17.

20 Ramon Llull, Llibre de vertuts e pecats, en Nova Edició de les obres de Ramon Llull, I, Palma de Mallorca, Moll, 1990, p. 10. 
liana, como una fuente fortuita que sirve, en ocasiones, para acabar de otorgar un sentido, ya no completo, sino menos heterodoxo para las mentalidades de la época.

Con el Pantocrátor acariciamos la materialización de las escrituras bíblicas, pues su representación sigue textualmente los siguientes pasajes de la Biblia: «Dominus caelum sedis meae et terra scabillum pedum meorum quae ista domus quam aedificabitis mihi et quis iste locus quietis meae» -Is. 66,1-; «ego autem dico uobis non iurare omnino neque per caelum quia thronus Dei est neque per terram quia scabillum est pedum eius neque per.Hierosolymam quia ciuitas est:magni Regis» -Mat. 5, 34-35-; «et dixit mihi factum est ego sum Alpha et Omega initium et finis ego sitienti dabo de fonte aquae:uiuae gratis»; - Apoc: 21,6 ; «ego Alpha et Omega primus et nouissimus principium et finis»-Apoc. 22,13- Ello es un claro ejemplo de la importancia que las sacras escrituras tienen respecto a las representaciones artísticas, puesto que una cita textual de la Biblia sirve para crear un elemento iconográfico tan importante en el mundo cristiano medievál como es la representación de Cristo triunfante, sentado, con los Evangelios en la mano izquierda y en actitud de bendecir. Esta manera de proceder consistiría en tomar al pie de la letra una referencia bíblica hasta plasmarla de forma material mediante recursos artísticos, mientras que en Llull lo que hallamos es un discurso en el que se insieren citas bíblicas, ya no tanto para reforzar el texto, sino para justificar - como veremos- la propia idea anunciada o que se enunciará a continuación. Entendemos, pues, la dificultad de interpretación que supone el Ars luliana en la época escolástica, ya que en la sociedad medieval el sistema luliano parece que no tiene cabida, ya no tanto por ser, o no, un «Arte alternativa», sino por la peligrosidad de ser tachado de sistema herético al relegar, de forma tan tajante, la autoridad bíblica. ${ }^{21}$

\section{LA AUCTORITAS BÍBLICA EN LA SEGUNDA ETAPA}

Es significativo que el nuevo sistema de predicación luliana surja en un contexto como el de la consolidación del Ars en su fase ternaria (del 1290 hasta el 1308, aproximadamente), momento en el cual Llull ha vivido ya dos experiencias en París, ninguna de ellas nada alentadoras. Sea como sea, observamos una reorientación en cuanto a la predicación homilética en los textos lulianos y, por consiguiente, una aproximación a las fuentes bíblicas. Ya hemos dicho más arriba que esta aproximación resulta muy sui generis, pero serán obras como el Liber de praedicatione (1304) la Summa sermonum (1312-1313) y el Ars abbreuiata praedicandi (1313) las que darán la clave de la relación de Llull con la Biblia. ${ }^{22}$ Según Fernando Domínguez, «Llull al presentar un nuevo tipo de sermón no pretende descalificar todo el programa homilético en boga sino ofrecer una nueva sistematización, un nuevo tipo de organización del material homilético» ${ }^{23}$ Ramon Llull rompe con los principios del sermón cristiano que, por ejemplo, hallamos reflejados en la máxima «Omnis praedicatio debeat fieri de sacris Scripturis» ${ }^{24}$-que

21 Tras la muerte de Llull, su sistema será condenado por la Inquisición y concretamente por el inquisidor Nicolau Eymerich. Véase, Jordi Pardo Pastor, «El lulismo del trescientos», en Alexander Fidora \& José G. Higuera (edd.), Ramon Llull caballero de la fe. El arte luliana y su proyección en la Edad Media, Cuaderno de Anuario Filosófico-Servicio de Publicaciones de la Universidad de Navarra, 2001, pp. 111-127.

22 Cfr. Raimundus Lullus, Liber de praedicatione (=Raimvndi Lulli Opera Latina. Tomus III-IV. Op. lat. 118), Abraham Soria (ed.), (Corpus Christianorum. Continuatio Mediaeualis, LXXX), Turnhout, Brepols, 1961-1963; Id. Summa sermonum in Ciuitate Maioricensi annis MCCCXII-MCCCXIII composita (=Raimvndi Lulli Opera Latina. Tomus XV. Op. lat. 208), Fernando Dominguez y Abraham Soria (edd.), (Corpus Christianorum. Continuatio Mediaeualis, LXXVI), Turnhout, Brepols, 1987; e Íd. Ars abbreuiata praedicandi (=Raimvndi Lulli Opera Latina. Tomus XVIII. Op. lat. 208), Abraham Soria, Fernando Domínguez y Michel Senellart (edd.), (Corpus Christianorum. Continuatio Mediaeualis, LXXX, Turnhout, Brepols, 1991.

23 Fernando Domínguez Reboiras, «Introductio generalis»), Raimundi Lulli Opera Latina. Tomus XV, p. xxxv.

24 Humberto de Romans, De eruditione praedicatorum, J. J. Berthier (ed.), Opera omnia, Roma, 1889 , vol. II, p. 400. 
nos da a entender que toda predicación ha de tener como base las sagradas escrituras-, utilizando las argumentaciones de su propia Ars con un único fin: demostrar las verdades de la fe. Este fin se complementa con la idea de una predicación dirigida a fieles e infieles, estableciendo dos modos de predicación: el modo praedicatio per aucthoritates y el modo praedicatio per moralem philosophiam. El desechar la auctoritas es un camino para aligerar de atavíos pomposos y artificiosos la predicación, pues Llull considera que de la retórica se valen aquellos que no poseen la verdad; los poseedores de la verdad no necesitan los artilugios de la retórica para convencer. ${ }^{25}$

Concibámoslo como lo concibamos, una sola cosa es cierta: hay un incremento considerable de citas bíblicas - con respecto a textos anteriores- en las obras que están escritas entre 1304 y 1311, aunque ello no significa un cambio en el usus scribendi luliano, sino, quizá, un nuevo posicionamiento con respecto a la Biblia que se consolida en dos puntos: por un lado, en una nueva visión de la predicación cristiana que no toma los themata bíblicos, sino que se fundamenta en el Ars luliana; y, por otro, en una toma de contacto con estos mismos themata bíblicos, que son meras pièces justificatives para ratificar que el Ars luliana es un sistema que va más allá de la Biblia, utilizándose la misma Biblia como elemento poderdante para ir más allá de las palabras y sentencias que se encierran en las sagradas escrituras. ${ }^{26}$ Cómo no, en este entramado juega un significativo primer plano el hecho de presentar el Ars luliana como revelación, pues ello y la manipulación de las sentencias bíblicas elaborada por Llull sirven de salvaguarda a todo el sistema filosófico luliano.

Si analizamos las obras del período que hemos marcado (1304-1311), es más que notable que, por ejemplo, en el Liber de consilio (1304) tan sólo podamos señalar dos citas bíblicas: la ya tan mencionada «Nisi credideritis, non intelligetis» e Io. 21, 15-17, «Petre, si amas me, pasce oues meas [...] Domine, tu scis, quod ego amo te ${ }^{27}$ que ya hallábamos, también, en el Arbor scientiae - Arbor apostolicalis (I, lin. 67). Mucho más descubrimos en el Liber praedicationis contra Iudaeos (1305), con un total de cuarenta y tres citas bíblicas, tal y como indica la edición de Aloisius Madre, en las que no se omiten Is. 7, 9, Deut. 6, 5, Leu. 19, 18, y Ps. 13, I, que se incluían ya en el Arbor scientiae. ${ }^{28}$ Sorprende de nuevo el Ars compendiosa Dei (1308) con dieciséis citas bíblicas cuando la obra anterior, mucho menor frente a la presente, contenía casi tres veces más. En comparación con el Liber praedicationis contra Iudaeos, en el Ars compendiosa Dei se repiten: Gen. I, I — «In principio creauit Deus caelum et terram»-y de nuevo Is. 7, 9; y respecto con el Arbor scientiae, Mat. 22, 37 e Is. 7, 9 —esta

25 Véase, Fernando Domínguez, «El proyecto luliano de predicación cristiana», en F. Domínguez y J. Salas (edd), Constantes y fragmentos del pensamiento luliano. Actas del simposio sobre Ramon Llull en Trujillo, 17-20 septiembre 1994, Tübingen, Niemeyer, 1996, pp. 117-132.

26 La Doctrina pueril (Gret Schib [ed.], Barcelona, Barcino, Els Nostres Clàssics, 1972) declara: «No som en temps de miracles, cor la devoció de convertir lo món en los Apòstols que no és ara en lo món en què som; ne rahons fundades sobre actoritatz no reeben los infeels; donchs convivent és convertir los infeels lo Libre de demostracions e la Art de atrobar veritat, la qual lus sia mostrada, per tal que ab ella los combata hom lur intel-ligència, per ço que conègan e amen Déu» (p. 197). De ello colegimos que las razones son necesarias para entender los articula fidei y además el mejor método resulta el Ars luliana, puesto que «Homo principaliter no sit creatus ad se, nec per consequens ad habendum meritum per fidem, imo principaliter est creatus ad intelligendum, diligendum et recolendum Deum» (Raimundus Lullus, Disputatio eremitae et Raymundi super aliquibus dubiis questionibus Sententiarum Petri Lombardi, en Íd., Beati Raimundi Lulli Opera, Ivo Salzinger [ed.], vol. IV, Maguncia 1725, p. 229).

27 Raimundus Lullus, Liber de consilio (= Raimundi Lulli Opera latina. Tomus $X$. Op. lat. 115), Louis SalaMolins (ed.), (Corpus Christianorum. Continuatio Mediaeualis XXXVI), Turnhout, Brepols, 1982, III, lin. 519 y III, lin. 438, respectivamente.

28 Raimundus Lullus, Liber de praedicatione contra iudaeos (= Raimundi Lulli Opera latina. Tomus XII. Op. lat. 123), Aloisius Madre (ed.), (Corpus Christianorum. Continuatio Mediaeualis XXXVIII), Turnhout, Brepols, 1984, esp. III, lin. 447 y VI, lin. 443; I, lin. 648; III, lin. 424; y II, lin. 330, respectivamente. 
últimà, como ya hemos señalado, también en el Liber de consilio. ${ }^{29}$ En el Liber de demonstratione per aequiparantiam (1305), de nuevo, y solamente, Is. $7,9,{ }^{30}$ al igual que en el De ascensu et descensu intellectus (1305). ${ }^{31}$ Por otro lado, el Liber de fine (1305) cuenta con diecisiete citas de las sagradas escrituras, de las que vuelven a repetirse respecto a los otros: Gen. 18 - Liber de praedicatione contra Iudaeos (I, lin. 147)--, Ex. 3, 14 -Liber de praedicatione contra Iudaeos (II, lin. 565)-, Ps. 109,3 - Liber de praedicatione contra Iudaeos (I, lin. 21)_, Is. 7, 9, Mat. 22, 37 - Arbor apostolicalis (I, lin. 67) y Ars compendiosa Dei (XXVII, lin. 177) - y Mat. 25, 41 - Arbor apostolicalis (IV, lin. 191 y V, lin. 171)-, y, por último, Io. 21, 17 - Liber de consilio (III, lin. 519) - ${ }^{32}$ De nuevo en el De experientia realitatis (13081309) únicamente: Is. 7,9.33 En el Liber de fide sancta catholica (1310) once citas bíblicas de las que se repiten respecto a los otros: Deut:6, 5-Arbor apostolicalis (IV, lin. 13) y Liber praedicationis contra Iudaeos (I, lin. 648)-, Is. 66, 9 - Liber praedicationis contra Iudaeos (I, lin. 813 y lin. 946)-, Mat. 12, 30 - Liber de Fine (lin. 138-139)_, Marc. 12, 30 - Arbor apostolicalis (IV, lin. 14)- e Io. 20, 27-29 — Ars compendiosa Dei (XXX, lin. 1289 y lin. 1302).${ }^{34}$ En el Liber de.ente (1311) hallamos tan sólo Mat. 12, 30; 25, 12 y 28, 18-20; y Marc. 4, 9 y 16,15 , mientras que en la Vita coaetanea (1311) contamos con veintidós citas bíblicas. ${ }^{35}$ Del mismo año de 1311, el Liber natalis cuenta con cuarenta y seis citaciones bíblicas - de las que se repiten gran parte de las ya aludidas, aunque se amplía la relación de libros bíblicos, citando fragmentos de los Prouerbia, Canticum Canticorum y Sapientia, libros que aún no habían aparecido-, y el Liber contradictionis cuenta tan sólo con tres. ${ }^{36}$

A simple vista, nada más observar la enumeración de citas bíblicas que hemos espigado en los libros señalados, se deduce lo siguiente: hay una marcada proliferación de determinados pasajes bíblicos, que se repiten una y otra vez. Existe, por ello, una evidente intertextualidad entre la obra luliana, creándose un propio 'mundo intertextual' al que recurrir y que se alimenta, paralelamente, del 'mundo exterior' (en este caso de la Biblia). ${ }^{37}$ Sin embargo, el

29 Raimundus Lullus, Ars compendiosa Dei (= Raimundi Lulli Opera latina. Tomus XIII. Op. lat. 134), Manuel Bauzà Ochogavía (ed.), (Corpus Christianorum. Continuatio Mediaeualis XXXIX), Turnhout, Brepols, 1985, esp. XXX, II, lin. 838 y 1025; XXIX, lin. 392 y XXX, II, lin. 1192; y XXVII, lin. 117, respectivamente.

30 Raimundus Lullus, Liber de demostratione per aequiparantiam (= Raimundi Lulli Opera latina. Tomus $L X$. Op. lat. 121), Aloisius Madre (ed.), (Corpus Christianorum. Continuatio Mediaeualis XXXV), Turnhout, Brepols, 1981, lin. 109.

31 Raimundus Lullus, De ascensus et descensus intellectus (= Raimundi Lulli Opera latina. Tomus IX. Op. lat. 121), Aloisius Madre (ed.), (Corpus Christianorum. Continuatio Mediaeualis XXXV), Turnhout, Brepols, 1981, esp. Ix, lin. 9.

32 Raimundus Lullus, Liber de fine (= Raimundi Lulli Opera latina. Tomus IX. Op. lat. 121), Aloisius Madre (ed.), (Corpus Christianorum. Continuatio Mediaeualis XXXV), Turnhout, Brepols, 1981, esp. lin. 252; lin. 122; lin. 253 ; lin. 235; lin. $140-141$; lin. 663-664; y lin. 24, respectivamente.

33 Raimundus Lullus, De experientia realitatis (= Raimundi Lulli Opera latina. Tomus XI. Op. lat. 138), Charles Lohr (ed.), (Corpus Christianorum. Continuatio Mediaeualis XXXVII), Turnhout, Brepols, 1983, esp. vII, lin. 119.

34 . Raimundus Lullus, Liber de fide sancta catholica (= Raimundi Lulli Opera latina. Tomus VI. Op. lat. 165), Helmut Riedlinger (ed.), (Corpus Christianorum. Continuatio Mediaeualis XXXIII), Turnhout, Brepols, 1978, esp. lin. 397; lin. 326; lin. 399; lin. 397; lin. 391; lin. 394, respectivamente.

35 Raimundus Lullus, Liber de ente/Vita coaetanea (= Raimundi Lulli Opera latina. Tomus VII. Op. lat. 188/189), Hermogenes Harada (ed.), (Corpus Christianorum. Continuatio Mediaeualis XXXIV), Turnhout, Brepols, 1980.

36 Raimundus Lullus, Liber natalis/Liber contradictionis (= Raimundi Lulli Opera latina. Tomus VI.I Op. lat. 169/171), Hermogenes Harada (ed.), (Corpus Christianorum. Continuatio Mediaeualis XXXII), Turnhout, Brepols, 1975 .

37 Asimismo, «un texto [en este caso entiéndase la obra luliana] representa una realidad extralingüística, cuya existencia pertenece al mundo real o a uno de los mundos posibles [...]) (José Enrique Martínez Fernández, La intertextualidad literaria, Madrid, Cátedra, 2001, p. 24). Pero, a nuestro parecer, esta realidad expresada no es unívoca, sino que, más bien, se relaciona con otro conjunto de realidades que se hallan a su alrededor. El mundo particular que se expresa en un determinado texto (ya sean estas expresiones verdaderas, ilusorias, erróneas...) fomenta 
hecho de crear una realidad 'alternativa' no puede realizarse, sin más, mediante la propia realidad, se precisa, de forma sine qua non, otra realidad o mundo del que retroalimentarse. La Biblia, sin duda, se erige como la fuente primordial suministradora, pese a sorprender la precisión con que Ramon Llull acude a la auctoritas, localizando, en todo momento, la cita clave para la interpretación de su Ars. Más arriba, hemos mencionado los florilegios o compilaciones de textos para ser memorizados, que habilitaban a encontrar con facilidad pasajes buscados y que servían, a su vez, como cuaderno de sentencias. Ante esto último, podemos aventurar dos perspectivas tales como la posibilidad de que Llull hubiera confeccionado un florilegio personal, el cual contenía las sentencias bíblicas que tanto repite y que dan sustento a su sistema, o la posibilidad de que Llull leyera algún florilegio usual en la época.

De esta suerte, el alimentarse de las auctoritates bíblicas, como hemos comprobado, no es unidireccional, ni constante, pues se observa que, en numerosas ocasiones, una obra es adornada con una o dos citas bíblicas —como el caso de los Liber de consilio, Liber de demonstratione per aequiparantiam, De ascensu et descensu intellectus, De experientia realitatis, y Liber contradictionis-, repitiéndose, sin excepción, en estas obras ahora señaladas, «Nisi credideritis, non intelligetis» Is. 7, 9. Este precepto intertextual apunta, en primer lugar, la deliberación de Llull al citar la Biblia, y, en segundo lugar, la continuación del mundo luliano. En definitiva, Ramon Elull está reinterpretando estas citas bíblicas desde la representación de su Ars, concluyendo, en la expresión de su propio pensamiento, en una hermenéutica luliana. Una hermenéutica que se vuelve a la Biblia y que constituye una nueva exégesis bíblica.

Si tomamos las tres citaciones más repetidas, tendríamos - Is. 7, 9-; «Dilige dominum Deum tuum ex toto corde tuo, et ex omni mente tua, et ex omnibus uiribus tuis» - Deut. 6, 5; Mat. 22, 37-; y «Petre, si diligis me, pasce oues meas»-Io. 21, 17. Estas tres citaciones tan copiadas cargan de significado apostólico la obra luliana, ya no tan sólo por el contenido que de ellas deducimos, sino, también, por la firmeza con que éstas aparecen y por la relación que se establece entre ellas y la misión apologética luliana. Si volvemos a los tres puntos expuestos más arriba con los que se resume el ideario luliano, tenemos: 1) el deseo de convertir a infieles e incrédulos a la fe católica no se puede realizar sin la fe, puesto que ésta es la condición previa al conocimiento -Is. 7, 9; 2) la intención de escribir el mejor libro del mundo para la conversión del infiel y poner en peligro la vida para dar a conocer la verdadera fe, se realiza por amor a Dios - Deut. 6, 5; Mat. 22, 37; y 3) el hecho de crear monasterios para la preparación de misioneros que lleven la tarea de convertir al infiel radica en un deseo de apacentar espiritualmente con más y mejores adeptos la cristiandad - Io. 21, 17. Ciertamente, esta intertextualidad o citación ad nauseam de estas autoridades bíblicas no supone un hecho casual o gratuito, pues mediante la Biblia lo que Llull está realizando es una explicación de la misión divina a la que se ha encomendado. Debemos ver, forzosamente, en este continuo y específico citar de las tres auctoritates bíblicas enumeradas un deseo de reforzar hermenéuticamente, con una sutilidad inigualable, los fines que Llull concibió para servir y ser agradable a Cristo, tras su inflamación de amor por Él.

la creación de otros mundos paralelos. Esta última idea puede vincularse con la cuestión kantiana que rechaza que los mundos paralelos de los que hablamos surjan de un mismo material, es decir, de un mismo mundo. Así pues, la construcción de mundos parte de mundos preexistentes, pero no de un mundo preexistente único. Crear mundos consiste en separar y en conjuntar (en ocasiones, al mismo tiempo) determinados elementos que conforman un mundo o más de uno. Es posible que diversas unidades pertenezcan a un mismo mundo, cruzándose con unidades de otros y produciendo un resultado diferente (aunque no siempre, ya que «no procedemos a crear un mundo nuevo cada vez que separamos dos cosas o cuando volvemos a unir otras, esta vez de manera diferente» [Nelson Goodman, Maneras de hacer mundos, Visor, Madrid, 1990, p. 26]). Los mundos pueden diferenciarse entre sí dependiendo de su propia naturaleza y de sus propios géneros y, a su vez, pueden distinguirse elementos que forman un mismo mundo, siendo ejecuciones de una misma obra, ya que se atienen a una iđéntica partitura, es decir, a una símil función primordial del mundo. Aunque, no es tanto que los géneros de un mundo resulten ausentes en otro, sino que si bien también están en él no suponen los rasgos distintivos predominantes de ese mundo. Estos rasgos distintivos de cada uno de los mundos tienen mucho que ver con el énfasis de cada uno de los autores que crean ese mundo. 
Tomando el orden que hemos establecido en el párrafo anterior, hay en estas citaciones un segundo plano, quizá, más explícito. En primer lugar, con Is. 7, 9, Llull está afirmando que si no conseguimos comprender la esencia de la divinidad mediante la experiencia,

[...] semper ad fidem recursum haberemus; et sic fides remanet in habitu. Etiam Isaias dixit: Nisis credideritis, non intelligetis. Quasi dicat: Si credimus, hoc quod credimus, mediante fide intelligere possumus. ${ }^{38}$

El sentido radica en que mediante la experiencia, mediante la razón, se busca el conocimiento de la esencia de Dios, aunque es la fe quien dicta los pasos de la razón, apoyándose fe en razón y convirtiéndose la fe en verdadera y expansiva. ${ }^{39}$ Del mismo modo lo expresa en el Arbor scientiae:

Sicut homo, qui credit, ut intelligere possit, et non posset intelligere illud obiectum, si prius in illo non crediderit; et hoc Isaias propheta significauit, in quantum dixit, si non creditis, non intelligitis. Et ideo in illa consequentia meritum restauratur per fidem. ${ }^{40}$

De esto último, podemos colegir que Ramon Llull toma dicha cita bíblica para prorrogarse, más adelante en el texto, con el verdadero significado que para él mismo posee dicha citación y para afirmar luego que si bien 'debemos creer, para entender', aquellos que no se hallan en la fe cristiana necesitan rationes necessariae para creer, pues: «Quoniam infideles dicunt: Nolumus dimittere fidem pro fide aut credere pro credere; sed bene credere pro intelligere dimittemus». ${ }^{41}$ En este sentido, como apunta Eusebi Colomer, «[...] l'esforç dialèctic del pensament no tendeix a abolir la fe, sino a trobar a l'interior de la fe un dimensió d'intel-ligència $[\ldots] \gg{ }^{42}$ Es aqui palpable que el planteamiento que Llull propone, en primer lugar, presenta la fe, mediante la cita de Is. 7, 9, como un elemento que, de por sí, sirve para la iniciación en el conocimiento de Dios, resultando, de ello, que al hombre le es imposible agotar el significado de la fe, puesto que la fe se mantiene en un plano superior, e ilumina al entendimiento cuando éste intenta comprender las alturas que la fe ilumina. Sin embargo, Llull esquiva las auctoritates, pues entiende que la demostración de la esencia divina debe realizarse mediante el conocimiento, es decir, a partir de la inteligencia, que ayudada por la fe alcanzará el fin último: el conocimiento de Dios. Por dicho motivo, Llull lo que pretende no es hacer que los infieles dejen una creencia por otra, sino, más bien, que abandonen un 'creer' por un 'entender'. Ramon Llull propone una nueva demostración, que prescinde de la demostración propter quid y propter quia, basada en la aequiparantia de los elementos con los que se establece una co-

38 Raimundus Lullus, Liber de demonstratione per aequiparantiam, op. cit., lin. 109.

39 Véase el texto que Llull escribe pocas líneas más arriba de la cita de Is. 7, 9: «Iterum dicimus, quod si aliquis diceret, quod ad probationem trinitatis fides destrueretur, sicut scriptum est, quod fides non habet meritum, cui praebet humana ratio experimentum, ad hoc respondemus, quod Iesus Christus Dominus Deus noster certificauit beatum Thomam, quando uoluit, quod per experientiam probaret fidei ueritatem, dum posuit manum suam in uulnere lateris eius. Et tamen hoc Iesus Christus non sustinuisset, si propter hoc beatus Thomas suum meritum amisisset; etiam quia experimentum non fit de spiritualibus, sed de corporalibus solum. Similiter nos non sumus creati maxime ad meritum nostrum, sed ad cognoscendum et diligendum et honorandum dominum Deum nostrum» (Raimundus Lullus, Liber de demonstratione per aequiparantiam, op. cit., lin.95-106).

40 Raimundus Lullus, Arbor scientiae, op. cit., Arbor quaestionalis, V, lin. 1912-1916.

41 Raimundus Lullus, Liber de demonstratione per aequiparantiam, op. cit., lin. 120-122.

42 Eusebi Colomer, «El diàleg interreligiós en Ramon Llull», en El pensament als països catalans durant l'edat mitjana $i$ el renaixement, Institut d'Estudis Catalans-Publicacions de l'Abadia de Montserrat, Barcelona, 1997, p. 147. 
rrespondencia (per aequalia), con una finalidad: equiparar razón y fe, filosofía y teología para llegar a un conocimiento pleno de la divinidad. ${ }^{43}$

Así pues, ya a punto de finalizar la introducción al Liber de demonstratione per aequiparantiam, Llull introduce esta sentencia bíblica (Is. 7,9), junto con la referencia a Tomás y Jesucristo- que sirve para justificar las tres distinciones del texto que vienen a continuación (I, De distinctione in diuinis; II, Quod sunt tres personae; III, In qua probatur, quod diuinae personae sunt Pater et Filius et Spiritus sanctus). A este modo de ver las cosas, Llull ha eliminado cualquier visión herética, pues si bien en su obra está aportando una visión que va más allá de la Biblia para dar a conocer la fe católica al infiel, es, eso sí, una visión que parte de la Biblia misma. El planteamiento que se establece de estas consideraciones es el que sigue: mediante la metafisica conocemos la Primera Causa, Dios, y, gracias a la teología, el acto racional que hemos alcanzado filosóficamente, nos conducirá, por medio de la fe, hacia ese ascensus tan anhelado. En Llull se produce una especie de camino circular, de eterno retorno, entre la fe y la razón. Se produce un camino entre los dos grados de conocimientos que Llull distinguía: scientia positiva per credere y scientia demonstratiua; y, mediante la demonstratio per hypothesim, Llull intenta avanzar de una a la otra, subministrando la suficiente razón a una creencia o a una opinión. ${ }^{44}$ De tal modo, «Nisi credideritis, non intelligetis» (Is. 7,9) sirve a Llull para distinguir entre scientia positiva per credere y scientia demonstratiua.

Con todo, debemos remarcar que Llull utiliza la Vetus latina al citar este pasaje de Is. 7 , 9, puesto que en su sistema apologético no hay cabida a una fe que se eriga como finalidad primera del intelecto del hombre y esta versión de la Biblia le proporciona el sentido conciliador entre fe y razón que está buscando, ya que la versión de san Jerónimo es un tanto devastadora: «si non credideritis non permanebitis» - Is. $7,9 .{ }^{45}$ La Vulgata no da mucha opción: debemos creer si queremos permanecer, es decir, el hecho de existir no viene dado por un factor como es el hecho de pensar, sino por la fe. Sin lugar a dudas, Llull debe excluir esta versión de san Jerónimo, porque desmantela su proyecto apologético.

Frente a Deut. 6, 5 y Mat. 22, 37, el Liber de fine ofrece un pasaje muy interesante en el que podemos observar cómo Llull manipula las citas bíblicas buscando el sentido que quiere aportar al texto:

Et iam dicerunt apostoli Iesu Christo (Luc. 22, 38): ecce, hic duos gladios. Et ipse respondit: Sufficiunt. Et per hoc fuit figuratum, quod bellare deberetis cum praedicatione et cum armis contra homines infideles. Et nescitis uos, quod Christus dixit (Matth. 12, 30): Qui non est mecum, contra me est? Et quod ipse praecepit dictum negotium figuratiue, in quantum dixit (Matth. 22, 37): Dilige Dominum,Deum tuum, ex toto corde tuo, etc. $?^{46}$

Si la máxima premisa del cristiano es 'amar a Dios con todo el corazón, el alma y la mente', no se incurre en ninguna atrocidad al querer desatar la cruzada contra los musulma-

43 Esta demonstratio per aequiparantiam se halla subdividida en tres modos: a) modo de igualdad de la razón entitativa, en tanto que hay una igualdad entre Bondad, Inteligencia, Ciencia, etc., divinas; b) modo de igualdad entre potencia y acto; y c) modo de igualdad entre los actos, como en la igualdad de las razones entendientes de Dios. Cfr. Miguel Cruz Hernández, El pensamiento de Ramon Llull, op. cit., p. 119. Por otro lado, Cruz Hernández denomina la demonstratio per aequiparantiam como un «modo de demostración "por extrapolación" y no por analogía, o igualdad, porque Ramon Lull niega rotundamente la predicación analógica [...] (ibidem).

44 Véase, Fernando Domínguez Reboiras, «Geometría, filosofia, teologia y Arte. En torno a la obra Principia philosophiae de Ramon Llull», SL 35 (1995), pp. 3-29, esp. 3-9.

45 Vnde subjungitur: Si non credideritis, non permanebitis (Is. 7, 9), ut Symmachus transtulit, id est, et vos non permanebitis in regno uestro, sed in captiuitatem ducemini, sustinentes eorum poenas, quorum imitati estis infidelitatem. Vel certe juxta LXX, non intelligetis. Et est sensus: quia quae Dominus dicit futura, non creditis, intelligentiam non habebitis (Hieronymus Stridonensis, Commentaria in Isaiam, PL xxIv, col. 104D).

46 Raimundus Lullus, Liber de fine, op. cit., lin. 134-141. 
nes, pues 'quien no está en Cristo, está contra Él'. Esta citación de la Biblia se erige como el elemento que evidencia que todo aquello que se propone en el Liber de fine, puede reinterpetrarse a partir de las sagradas escrituras y bajo el sistema apostólico luliano, manteniendo, de nuevo, una cita de la Biblia que lleva a una interpretación que se halla más allá, pero que parte de las mismas letras divinas. En este aparte, sin embargo, no se apela a una interpretación filosófica basada en la dualidad entre fe y razón, sino que, más bien, el texto es muy directo. ${ }^{47}$

El Liber de fine incide en la idea del bellator rex y en la inmediata adquisición de Granada y Tierra Santa, y fue dedicado por Llull a Jaume II de Aragón, convenciéndolo para que lo presentara al Papa Clemente V. No hay más que diecisiete citas bíblicas en este opúsculo luliano, pese a ser una obra que va encaminada al Papa, aunque se presenta la suficiente carga homilética en el texto al preguntar retóricamente, «¿Y no sabéis vos que Cristo dijo: Quién no está conmigo, está contra mi?», y terminar, rotundamente, con «Ama al Señor, Dios tuyo, con todo tu corazón». Sin duda, este párrafo es una prueba de hecho necesaria (tekméria) dirigida a Clemente $\mathrm{V}$ y que se ve complementada con la citación de Io. 21, 17-《Petre, si diligis me, pasce oues meas»- en el exordio. ${ }^{48}$ Llull expone sus argumentos de forma muy clara: en primer lugar, sugiere que el Papa, al igual que Pedro encomendado por Jesucristo, y al igual que todo buen cristiano que se precie de ello, tiene la tarea de apacentar a los cristianos y procurar que todos aquellos que no han hallado, aún, la verdadera fe la alcancen, pues la conversión del infiel es un camino de amor al Señor; y, en segundo lugar, afirma que puesto que se debe convertir a los infieles, aquellos que no quieren oír las razones de la verdadera fe, están en contra de Cristo, y deben ser combatidos, ya que el principal precepto de la fe de Cristo es amar a Dios sobre todas las cosas. De nuevo, las citas bíblicas son meras piezas justificativas que intentan incidir en el pathos del lector para argumentar la perspectiva luliana (en estos momentos a favor de la cruzada), no sólo desde el punto de vista del Ars, sino, también, desde el punto de vista de la escolástica cristiana al incluir fragmentos de la Biblia.

\section{CONCLUSIO}

Un sistema como el que Ramon propone se desvía, considerablemente, de las directrices que la escolástica marcaba. Ya en vida, Ramon Llull tuvo sus venturas y desventuras con su sistema filosófico, teniendo que modificarlo, en ocasiones, para adecuarlo a los teólogos cristianos debido a la fragilidad del entendimiento humano. Este cambio en el Ars luliana de un sistema cuaternario a un sistema ternario, obedece a exigencias del guión, es decir, Llull debe simplificar su Ars para que sus contemporáneos puedan comprenderle mejor. Por ello, podríamos aventurar una posible adecuación del Ars frente a la Biblia debido a estos motivos externos, a saber, un modo de justificar la obra luliana a partir de una determinada sentencia bíblica, sirviendo esta adecuación como método justificativo para introducir el Ars luliana en la mentalidad Trecentista escolástica, pues Llull, tras su primera estancia en París, es consciente de que mediante su modus loquendi arabicus no puede dar a entender su Ars. Llull utiliza la Biblia como herramienta positiva, ya que no está razonando mediante auctoritates, sino que, más bien, la auctoritas es el pretexto que lleva a la argumentación luliana.

47 A este tenor, y parafraseando al Llull poético del Desconhort, a los musulmanes hay que combatirlos con el hierro, la madera y el argumento verdadero («[...] ab ferre e fust e ab ver argument»). Drost Beattie, Iberia and the Mediterranean World of the Middle Ages. Studies in Honor of Robert I. Burns S. J., Larry J. Simon (ed.), Leiden/New York, E. J. Brill, 1995, pp. 113-129, aquí pp. 113-116) afirma que no hay contradicción entre los conceptos de misión y de cruzada, pues para Llull la fuerza es un recurso extremo que debe estar siempre acompañado de la evangelización. Cfr. supra nota 14 .

48 Raimundus Lullus, Liber de fine, op. cit, lin. 24. 
Ramon no utiliza la auctoritas, sin más, como elemento argumentativo, sino que se vale de la cita para fundamentar y salvaguardar su obra y su ideario. Partiendo de una hermenéutica bíblica que se basa en su propia figura de Iluminado y en el ideal caballeresco a lo divino, pasa a un período en el que se toman citas bíblicas - aunque escasas y de forma repetitivapara justificar que un sistema como el Ars Iuliana que va más allá de la Biblia, parte, asimismo, de las sagradas escrituras. Se deduce que entre las dos primeras etapas que hemos propuesto no existen diferencias palpables, hay un incremento o intensificación en citas bíblicas, ya que si en las primeras obras asistimos a una hermenéutica del texto bíblico, a una adecuación de las enseñanzas bíblicas traspuestas al Ars luliana, en esta segunda etapa, dicha hermenéutica: sufre una modificación de modo, pero no de forma. En esta segunda etapa, la expresión del pensamiento luliano se ve autorizada por una sentencia bíblica, aunque se sigue constituyendo como método de interpretación general basado en el prisma luliano. La tercera etapa, como apunta Reinhardt, carecerá, de forma incomprensible, de cualquier cita bíblica, dejando atrás la hermenéutica aquí expuesta.

La etapa reseñada en el presente trabajo ostenta una singular intertextualidad luliana, que no es otra cosa que la adecuación de un mundo extraliterario personal —el que Llull propone en sus escritos-, que conduce a una aserción incuestionable: sin «amància», no hay «ciència». No obstante, la creación de este mundo se fundamenta en el rol que el mundo escolástico propone, aunque con una perspectiva y originalidad que va más allá y que sitúan el Ars luliana, ya no sé si como «Arte alternativa», o como sistema o mundo independiente. Ciertamente, aunque dicho mundo no posea los rasgos distintivos predominantes del otro mundo (en este caso, el sistema escolástico), no se encuentran éstos ausentes y, dependiendo del énfasis con que se traten, dan razón de ser a la nueva visión luliana, pues en ellos se alimenta o de ellos se diferencia.

Jordi Pardo Pastor «ARCHIVIVM LVLLIANVM»-Universitat Autònoma de Barcelona Departament de Ciències de l'Antiguitat i de l'Edat Mitjana Edifici B 08193 Bellaterra (Barcelona) jordi.pardo@campus.uab.es 